Editorial

\title{
Recent advances in location analysis
}

This special issue is dedicated to recent advances in location analysis. As described by the EURO Working Group on Locational Analysis (EWGLA), the subjects of interest within location analysis are of many kinds, centering on the optimal choice of locations for one or more facilities, within any framework (the classical settings being discrete, network, and planar) in a way that optimizes a certain objective such as minimizing transportation costs, providing equitable service to customers, capturing the largest market share, etc. Many other fields have direct connections with location analysis, either by subject such as transportation and routing, supply chain management, environmental studies, layout and design, data and cluster analysis, or as techniques, like mathematical (linear, integer, non-linear, convex, global, etc.) programming, multi-criteria analysis, approximation theory, computational geometry, statistics, etc. The aim of this special issue was to collect high quality papers that address recent advances in theory, practice, and application.

The idea for this special issue emerged during the 20th EWGLA Meeting, which took place in Ankara, Turkey on April 17-19, 2013, organized by the editors. There were a total of 53 presentations and participants from 16 different countries in this meeting. The articles in this special issue, however, are not restricted to the presentations made in this meeting.

We received a total of 64 submissions addressing various problems in the context of location analysis. At the end of the reviewing process, 19 papers were accepted to be published. Below, we shortly outline the content and contribution of the 19 papers that were selected for publication in this special issue.

Quevedo-Orozco and Ríos-Mercado propose a new metaheuristic for the capacitated vertex $p$-center problem. This metaheuristic integrates a greedy randomized procedure with adaptive probabilistic selection in the construction phase and an iterated greedy local search with a variable neighborhood descent in the local search phase.

Albareda-Sambola, Hinojosa, Marín, and Puerto present an extension to the $p$-center problem considering that centers can fail. They introduced the $p$-next center problem and developed several different integer programming formulations with strengthening methods based on valid inequalities and variable fixing.

Zhao and Verter address a location-routing problem involving the collection, transportation, and disposal of used oil. The problem involves both location decisions on facilities and routing decisions on the circular trips starting and ending at the storage facilities and on the one-way trip from storage facilities to integrated facilities. They propose a bi-objective model which incorporates risk coefficients computed by integrating the box model and the Gaussian plume model for environmental risk assessment.

Huang, Wang, Batta, and Nagi focus on the problem of selecting sites and deciding on the storage capacities of distribution centers while assuming that shipment requests from a plant to a customer arrive randomly. The authors employ an $\mathrm{M} / \mathrm{G} / \mathrm{c}$ queuing model for the storage operations at each distribution center.

Farham, Süral, and Iyigun study the single facility restricted Weber problem in congested regions with entry and exit points. They developed an evolutionary algorithm modified with variable neighborhood search to solve this problem.

Yu and Lin consider the open location-routing problem motivated from the third party logistics companies. The authors develop a simulated annealing based heuristic which perform well on the benchmark instances.

Verma and Gaukler study facility location in humanitarian supply chains by considering increased damage probabilities for facilities with increasing proximity to the epi-center of the disaster. They provide a detailed case study using large-scale emergencies caused by an earthquake in California to demonstrate the performance of the proposed deterministic and stochastic models.

Halper, Raghavan, and Sahin present a new formulation and propose local search heuristics for the mobile facility location problem in which one seeks to relocate (or move) a set of existing facilities and assign clients to these facilities so that the sum of facility movement costs and the client travel costs is minimized.

Damgacioglu, Dinler, Ozdemirel, and Iyigun propose a mathematical formulation and a genetic algorithm for the uncapacitated single allocation planar hub location problem. They present the first results on the $\mathrm{CAB}$ and $\mathrm{AP}$ data sets from the literature for a planar hub location problem and compare these with the solutions of the discrete problem.

Dinler, Tural, and Iyigun present a continuous multi-facility location allocation problem where the demanding entities are regions in the plane, together with several heuristics to solve the resulting location problem.

Ventura, Hwang, and Kweon study the continuous location problem for a single refueling station on a tree network maximizing the traffic flow in round trips per day that can be covered by the station. They developed an exact polynomial algorithm for the solution of this problem.

Salman and Yücel focus on the locations of emergency response facilities in the pre-disaster stage while explicitly taking the vulnerability of the transport network into consideration. The authors apply their solution methodology to Istanbul's network, which is under a high earthquake risk. 
Sen, Krishnamoorthy, Rangaraj, and Narayanan study the problem of locating a very large database of files in an information network in a cost-efficient manner for an easy access to these files. They propose two modeling approaches and an exact Benders decomposition algorithm.

Drezner, Brimberg, Mladenović, and Salhi investigate different heuristics for solving the $p$-median problem in the plane and develop three new approaches: a variant of variable neighborhood search, a genetic version, and a hybrid heuristic combining both.

Eiselt and Marianov introduce a generic cost-minimizing model for the landfill siting problem and survey studies that include applications of landfill location models using actual data.

Ruiz-Hernández, Delgado-Gómez, and López-Pascual address the problem of restructuring bank networks by resizing open branches in order to maintain a constant service level, while considering both, closing down and long term operation costs, and the presence of competitors, allowing for ceding market share whenever the restructuring costs are prohibitively expensive.

Wei and Murray study the problem of locating facilities in continuous space to maximize the coverage of regional demand. The authors present theoretical and empirical analysis of spatial representation approaches and potential errors that could result when these approaches are used in solving this problem.

Redondo, Fernández, Hervás, Arrondo, and Ortigosa consider a bi-objective competitive facility location problem and propose a new evolutionary multi-objective optimization algorithm for the solution of this problem.
Menezes and Huang compare the quality of a facility location resulting from voting and that of a centralized decision. They developed an upper bound for the ratio of the Weber objective function value evaluated at the Condorcet solution, to the optimal Weber value.

As detailed above, various facility location problems, arising from a number of applications, are introduced, modeled, and solved in this special issue.

Finally, we would like thank all of those who contributed to this special issue. In particular, all the authors for submitting and revising their work and all the reviewers for their valuable time and constructive criticism which certainly improved the quality of all the publications.

Sibel A. Alumur*

Department of Management Sciences, University of Waterloo, Waterloo, Ontario, Canada E-mail address: sibel.alumur@uwaterloo.ca

Bahar Y. Kara Department of Industrial Engineering, Bilkent University, Ankara, Turkey

E-mail address: bkara@bilkent.edu.tr 